\title{
On the role of the scale invariance and the independent components of natural scenes on the receptive fields of simple cells Néstor Parga* and José M Delgado
}

\author{
Address: Department of Theoretical Physics, Universidad Autónoma de Madrid, Madrid, Spain \\ Email: Néstor Parga* - nestor.parga@uam.es \\ * Corresponding author
}

from Eighteenth Annual Computational Neuroscience Meeting: CNS*2009

Berlin, Germany. 18-23 July 2009

Published: 13 July 2009

BMC Neuroscience 2009, 10(SuppI I):P357 doi:10.I I86/I47|-2202-I0-SI-P357

This abstract is available from: http://www.biomedcentral.com/I47I-2202/I0/SI/P357

(c) 2009 Parga and Delgado; licensee BioMed Central Ltd.

\section{Introduction}

Predicting the receptive fields (RFs) of visual neurons from the statistical properties of the natural stimuli requires, as a first step, to determine the relevant regularities of the visual world [1]. This program seems to be successful for retinal and LGN ganglion cells whose RFs have been explained [2] by using the scale invariance exhibited by the power spectrum of natural scenes. In spite of many efforts, modeling work has failed to predict the properties of the RFs of V1 simple cells [3]. This is partly due to the fact that: 1) the independent components of natural scenes appear in a nonlinear manner and 2) that statistical symmetries - which are strong regularities- such as scale invariance is more complex than the power behavior of the power spectrum $[4,5]$.

\section{Results}

Here we describe how these two issues are related and an adequate treatment of the statistical scale invariance of natural scenes leads to theoretical RFs with properties in agreement with experimental observations. In particular the model gives the region in the space of Gabor parameters where the Gabor fits of the RFs of the simple cells should be, in principle for any species. Comparison with existing data for three species confirms this prediction. The spatial phase distribution is species dependent and the model is in agreement with the observed differences.

\section{Conclusion}

By exploiting the scale invariance properties of natural images the model makes several predictions on distributions of the parameters of Gabor fits of V1 simple cells. The model can discriminate between properties of these cells in different species. Since it establishes a direct connection between the visual environment of a given species and the RFs of its simple cells, from a good knowledge of the first the model could determine more precisely their spatial structure and distributions.

\section{Acknowledgements}

We warmly thank A. Turiel for many helpful discussions and for help in some aspects of this work.

\section{References}

I. Barlow HB: Possible principles underlying the transformation of sensory messages. In Sensory Communication Edited by: Rosenblith W. Cambridge MA: MIT Press; 196I.

2. Attick J]: Could information theory provide an ecological theory of sensory processing? Network: Comput Neural Syst 1992, 3:2I3-25I.

3. Ringach D: Spatial stucture and symmetry of simple-cell receptive fields in macaque primary visual cortex. I Neurophysiol 2002, 88:455-463.

4. Turiel A, Mato G, Parga N, Nadal JP: The self-similarity properties of natural images resemble those of turbulent flows. Phys Rev Lett 1998, 80:1098-II0I.

5. Turiel A, Parga N: Multifractal wavelet filter of natural images. Phys Rev Lett 2000, 85:3325-3328. 